\title{
Wideband Impedance-varying $N$-way Wilkinson Power Divider/Combiner for RF Power Amplifiers
}

\author{
Osama Hussein ${ }^{1}$, Khair Al shamaileh ${ }^{2}$, Vijay Devabhaktuni ${ }^{1}$, and Peter Aaen ${ }^{3}$ \\ ${ }^{1}$ University of Toledo, OH, US, ${ }^{2}$ Purdue University Northwest, IN, US, ${ }^{3}$ University of Surrey, Guildford, UK
}

\begin{abstract}
We propose a compact wideband multi-way power divider/combiner with planar structure for applications to RF power amplifiers (PAs). Uniform transmission lines in the conventional divider are replaced with non-uniform transmission lines (NTLs), which are governed by a truncated Fourier series. An optimization-driven framework is employed in even-mode analysis to obtain the coefficients of the NTLs considering predefined operating bands, whereas three isolation resistors are optimized in the odd-mode analysis to achieve optimal isolation and output port matching over the design bandwidth. For verification purposes, a 4-10 GHz 3-way divider is simulated, and measured. Simulations and measurements are in close proximity and show input/output ports matching of better than $-10 \mathrm{~dB}$ and transmission of $-4.9 \pm 1 \mathrm{~dB}$ across the design bandwidth.
\end{abstract}

Index Terms - multi-way power divider, non-uniform transmission lines (NTLs), power amplifiers (PAs), power combiner, wideband Wilkinson power divider (WPD).

\section{INTRODUCTION}

Power dividers/combiners are widely used fundamental frontend components in a variety of microwave/millimeter wave systems, power amplifiers (PAs), mixers, and antenna feeding networks. PAs have applications that span wireless and radio communications equipment, phased array radar modules, unmanned aerial vehicles (UAVs), and electromagnetic compatibility (EMC) testing [1]-[5]. PAs can also be used in communication systems measurements such as tests of intermodulation, adjacent channel power, multi-tone, and high signal levels. However, PAs have limitations, especially for applications where a high $\mathrm{RF}$ amplification gain is required. To overcome this, a power combiner can be used to combine the individual powers from multiple lower power PAs. Such a network has the advantages of: 1) graceful degradation reliability characterized by the accommodation of the failure of a single PA without a total loss of the transceiver/transponder power [6], 2) it is less expensive and complex to manufacture moderate-power PAs than highpower PAs, and 3) lower power PAs can be biased to operate in the linear region, resulting in the reduction of the intermodulation distortion and undesired harmonics.

Many power dividers/combiners for large operating bandwidths, high efficiency, high isolation, and small size have been proposed in literature. The Wilkinson power divider (WPD) or combiner, proposed by E. Wilkinson in 1960 [7], is widely used in frontend microwave subsystems due to its ease of design and fabrication, high isolation between its ports, as well as perfect matching at all ports. Port isolation is widely regarded as an asset in RF power combining, as it can be used to suppress odd mode instability between the combined amplifiers [8]. This alone often explains the preference of Wilkinson type combiners over other dividers/combiners in microwave PA networks. Accordingly, broadening the operating bandwidth while maintaining the same desired electrical features are of utmost importance. A variety of designs have been proposed [9]-[13], however, the major drawbacks are the increased physical size, the use of reactive components, and the bulky $3 \mathrm{D}$ geometries.

In this paper, a general procedure to design and characterize a NTLs wideband multi-way planar Wilkinson power divider/ combiner is presented. The proposed device is modeled and simulated for equal-split division. Then, the input and output ports can be reversed to enable power combining rather than division. Thereafter, the phases of the transmission parameters are measured to verify in-phase functionality.

The article is organized as follows: Section II discusses the design procedure of the proposed wideband NTL multi-way WPD. Simulations and measurements of a wideband 3-way prototype is presented in Section III. Finally, conclusions are given in Section IV.

\section{MULTi-WAY WPD DESIGN}

A schematic diagram of the proposed wideband equal-split NTL divider is shown in Fig. 1. The $N$-way divider can be reduced to its equivalent 2-way model [14]. Hence, the evenodd mode analysis will be adopted.

To reduce the $N$-way divider to its equivalent 2 -way model illustrated in Fig. 2, the first branch is assumed to be the $n$-th branch with $1 / N$ fraction of total input power, whereas the other branch is in turn the sum of power ratios of the rest of the $N-1$ branches; or in other words, $(N-1) / N$.

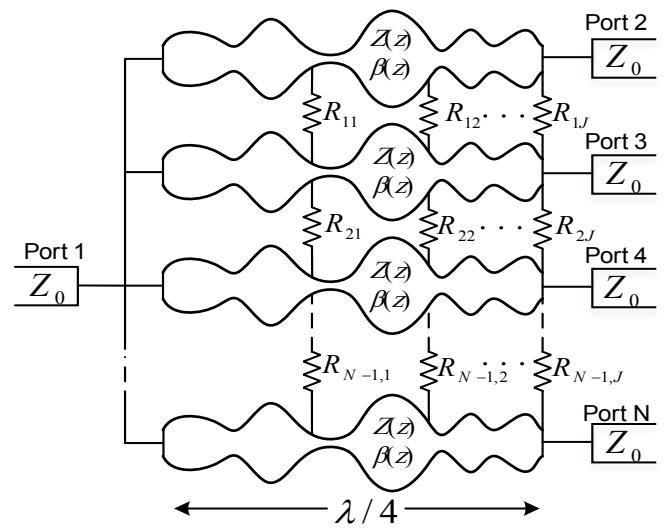

Fig. 1. A Schematic diagram of a wideband NTL $N$-way WPD. 


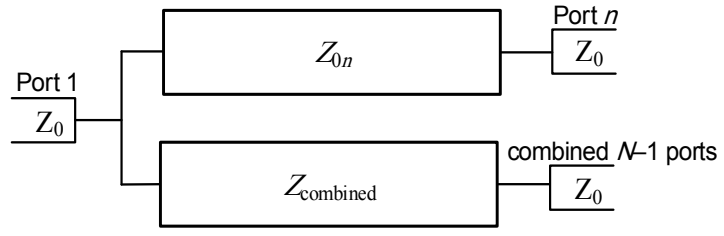

Fig. 2. A 2-way equivalent model of the $N$-way WPD.

Following the procedure presented in [14], $Z_{0 n}$ is found to be equal to $103 \Omega$. Once the characteristic impedance of the $n$ - $t h$ branch is determined, the process is iterated until the characteristic impedances of all the $N$ original branches are obtained. In this context, the equivalent 2 -way model is developed only once as the power is split equally between the $N$ output ports (i.e., same characteristic impedances $Z_{0 n}$ for all $N$ branches).

\section{A. Even Mode Analysis}

The even mode equivalent circuit for the proposed $N$-way divider is shown in Fig. 3. The NTL has a length $d$ with a varying characteristics impedance $Z(z)$ and propagation constant $\beta(z)$, and matches a source impedance $Z_{s}$ to a load impedance $Z_{l}$. The isolation resistors $R_{1 j} / 2(j=1,2,3)$ are terminated with an open circuit as a result of the symmetric excitation at the two equivalent output ports. To design the NTL, the magnitude of the reflection coefficient, $|\Gamma|$, should be zero (or very small) over the frequency range of interest. $|\Gamma|$ at the input port is expressed in terms of $Z^{e}{ }_{i n}$ shown in Fig. 3, where $Z^{e}{ }_{i n}$ is calculated after obtaining the $A B C D$ parameters of the NTL.

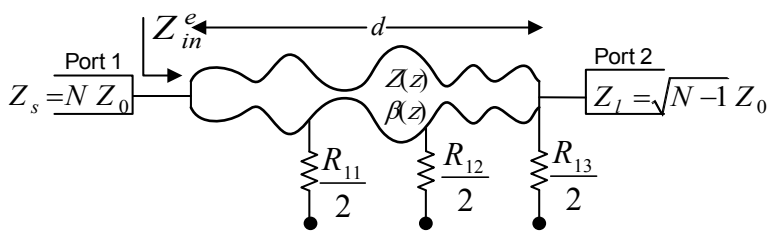

Fig. 3. 2-way equivalent even mode circuit of the proposed NTL $N$-way WPD.

The $A B C D$ parameters of the wideband matching NTL are obtained by subdividing it into $K=50$ uniform short segments each of length $\triangle z$. The $A B C D$ matrix of the whole NTL is obtained by multiplying the $A B C D$ parameters of each section as follows [15]:

$$
\left[\begin{array}{ll}
A & B \\
C & D
\end{array}\right]_{Z(z)}=\left[\begin{array}{cc}
A_{1} & B_{1} \\
C_{1} & D_{1}
\end{array}\right] \cdots\left[\begin{array}{cc}
A_{i} & B_{i} \\
C_{i} & D_{i}
\end{array}\right] \cdots\left[\begin{array}{cc}
A_{K} & B_{K} \\
C_{K} & D_{K}
\end{array}\right] .
$$

where the $A B C D$ parameters of the $i^{\text {th }}$ segment are [15] (assuming lossless transmission lines):

$$
\begin{gathered}
A_{i}=D_{i}=\cos (\Delta \theta), \\
B_{i}=Z^{2}((i-0.5) \Delta z) C_{i}=j Z((i-0.5) \Delta z) \sin (\Delta \theta), \\
i=1,2, \ldots, K, \\
\Delta \theta=\frac{2 \pi}{\lambda} \Delta z=\frac{2 \pi}{c} f \sqrt{\varepsilon_{\text {eff }}} \Delta z .
\end{gathered}
$$

where $c$ is the speed of light and $f$ is the center frequency of the design bandwidth. The effective dielectric constant, $\varepsilon_{\text {eff, }}$ of each section is calculated using the well-known microstrip line formulas [15]. Furthermore, the non-uniform impedance profile is governed by a truncated Fourier series that is expressed as [16]:

$$
Z(z)=Z_{0 n} \exp \left(c_{0}+\sum_{p=1}^{P}\left[a_{p} \cos \left(\frac{2 \pi p z}{d}\right)+b_{p} \sin \left(\frac{2 \pi p z}{d}\right)\right]\right) .
$$

where $Z_{0 n}=\left(Z_{s} Z_{l}\right)^{0.5}$, is the characteristic impedance of the WPD branch. Thus, an optimum designed NTL has its reflection coefficient magnitude over the frequency range of interest, with an increment of $\Delta f$ as close as possible to zero. Therefore, the optimum values of the Fourier coefficients can be obtained through minimizing the following error function [17]:

$$
\text { Error }_{\text {in }}=\max \left(E_{f_{1}}^{\text {in }}, \ldots, E_{f_{m}}^{\text {in }} \ldots, E_{f_{M}}^{\text {in }}\right) \text {. }
$$

where

$$
\begin{gathered}
E_{f_{m}}^{\text {in }}=\left|\Gamma_{i n}\left(f_{m}\right)\right|^{2} . \\
\Gamma_{i n}\left(f_{m}\right)=\frac{Z_{i n}^{e}\left(f_{m}\right)-Z_{s}}{Z_{i n}^{e}\left(f_{m}\right)+Z_{s}} . \\
Z_{i n}^{e}\left(f_{m}\right)=\frac{A\left(f_{m}\right) Z_{l}+B\left(f_{m}\right)}{C\left(f_{m}\right) Z_{l}+D\left(f_{m}\right)} .
\end{gathered}
$$

The resulting $Z(z)$ should be within reasonable fabrication tolerances to guarantee easy fabrication. As such, the following physical constraint is considered in the minimization of (4):

$$
Z_{\min } \leq Z(z) \leq Z_{\max }
$$

To find the values of the coefficients $\left(c_{0}, a_{p}, b_{p}\right)$ that minimize the bound-constrained nonlinear error function in (4) across the desired bandwidth, MATLAB function "fmincon.m" is used.

\section{B. Odd Mode Analysis}

Figure 4 shows the equivalent odd-mode circuit of the proposed divider which is used to obtain the optimum values of the isolation resistors that achieve acceptable output ports isolation and matching conditions. Due to the asymmetric excitation of the output ports, each $R_{1 j} / 2$ resistor will be terminated with a short circuit.

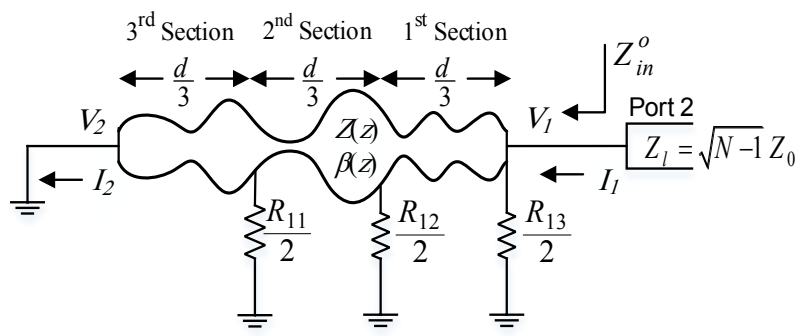

Fig. 4. 2-way equivalent odd mode circuit of the proposed NTL $\mathrm{N}$-way WPD.

After determining the optimum values of the Fourier series coefficients by following the procedure described previously, the NTL transformer is subdivided into 3 sections, and the $A B C D$ matrix for each section is calculated by employing (1) and (2). Then, the total $A B C D$ matrix of the whole network shown in Fig. 4 can be calculated as follows [17]: 


$$
\begin{aligned}
& {[A B C D]_{\text {Total }}=[A B C D]_{\frac{R_{13}}{2}} \cdot[A B C D]_{1 \text { st Section }} \cdot[A B C D]_{\frac{R_{12}}{2}} .} \\
& {[A B C D]_{2 \text { nd Section }} \cdot[A B C D]_{\frac{R_{11}}{2}} \cdot[A B C D]_{3 \text { rd Section }}}
\end{aligned}
$$

The locations of the resistors are distributed uniformly. Finally, as illustrated in Fig. 4, the following equation can be written:

$$
\left[\begin{array}{c}
V_{1} \\
I_{1}
\end{array}\right]=\left[\begin{array}{ll}
A & B \\
C & D
\end{array}\right]_{\text {Total }}\left[\begin{array}{c}
V_{2} \\
I_{2}
\end{array}\right] \text {. }
$$

Setting $V_{2}$ in (8) to zero, and solving for $V_{1} / I_{1}$, one obtains:

$$
\frac{V_{1}}{I_{1}}=\frac{B}{D}=Z_{\text {in }}^{o}
$$

For perfect output port matching, the following condition should be satisfied:

$$
\Gamma_{\text {out }}\left(f_{m}\right)=\frac{Z_{\text {in }}^{o}\left(f_{m}\right)-Z_{0}}{Z_{\text {in }}^{o}\left(f_{m}\right)+Z_{0}} .
$$

where $f_{m}(m=1,2, \ldots, M)$ denotes the frequencies at which (10) is calculated. In this context, $\Delta f$ is chosen to be $0.2 \mathrm{GHz}$. So, for a perfect output ports matching over the desired range, the following error should be minimized [17]:

$$
\text { Error }_{\text {out }}=\max \left(E_{f_{1}}^{\text {out }}, \ldots E_{f_{m}}^{\text {out }} \ldots E_{f_{M}}^{\text {out }}\right) \text {. }
$$

where

$$
E_{f_{m}}^{\text {out }}=\left|\Gamma_{\text {out }}\left(f_{m}\right)\right|^{2} .
$$

This optimization problem is solved keeping in mind that $R_{11}$, $R_{12}$, and $R_{13}$ are the optimization variables to be determined.

\section{SiMULATIONS AND MEASUREMENTS}

In this section, based on the design procedure presented in II, a design example of a 3-way equal split WPD with fractional bandwidth of $86 \%$ is simulated, fabricated and measured. The design example is carried out using RO4003C substrate with a thickness of $0.813 \mathrm{~mm}$, relative permittivity of 3.55 , and loss tangent of 0.0027 . The length of each NTL transformer of the proposed WPD is set to $10 \mathrm{~mm}$. The characteristic impedance of the conventional 3-way WPD arm, $Z_{0 n}$, is calculated in II and is found to be equal $103 \Omega$. Figure 5 shows a photograph of the fabricated power divider.

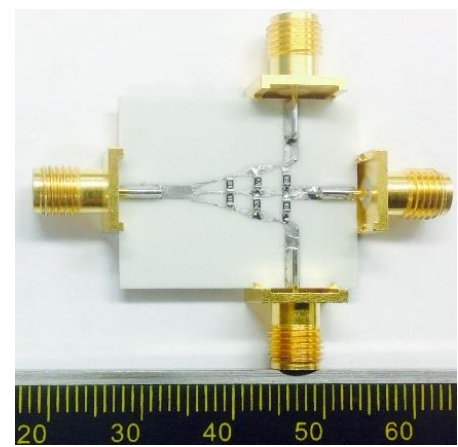

Fig. 5. Photograph of the fabricated 3-way WPD structure.
Simulations were obtained using HFSS, which is a full-wave EM simulator, whereas measurements were made with an HP 8720B vector network analyzer (VNA). Short-open-load-thru calibration was performed to the two ports of the VNA using a $3.5 \mathrm{~mm} \mathrm{HP} /$ Agilent 85052A mechanical calibration kit. First, port 1 of the VNA was connected to an open-circuit standard and calibrated. Then, it was connected to a short-circuit standard and calibrated. Finally, it was connected to a broadband $50 \Omega$ matched load for load calibration. The same process was repeated for port 2 of the VNA. Thru calibration was made by connecting the two VNA ports together to compensate for the losses due to cables and connectors.

After a two-port calibration was performed, input/output matching parameters of the proposed divider/combiner were measured by connecting VNA port 1 to the divider's port of interest, while terminating the remaining ports with a matched load. Transmission parameters were measured by connecting VNA port 1 to the input port of the proposed divider and VNA port 2 to one of the output ports; whereas the rest of the divider's ports were terminated with a matched load. Figures 6 and 7 illustrate the resulting simulated and measured scattering parameters.

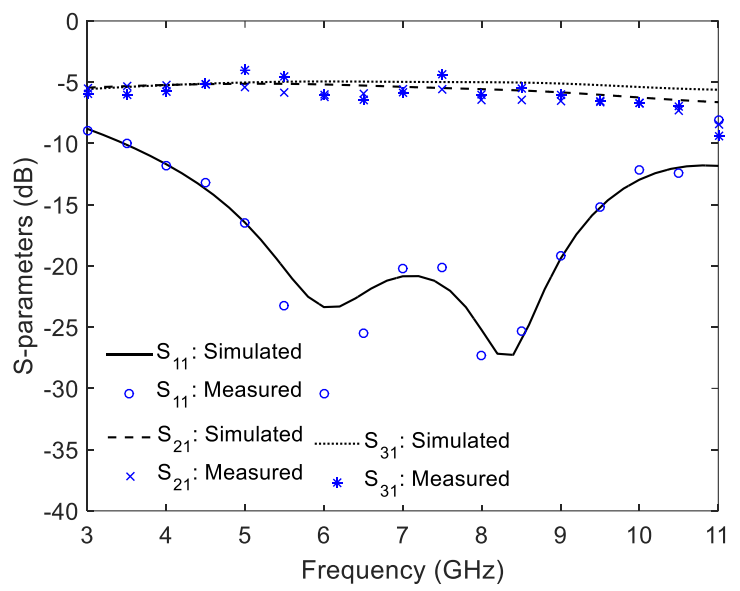

Fig. 6. Simulated and measured $S$-parameters of the proposed divider.

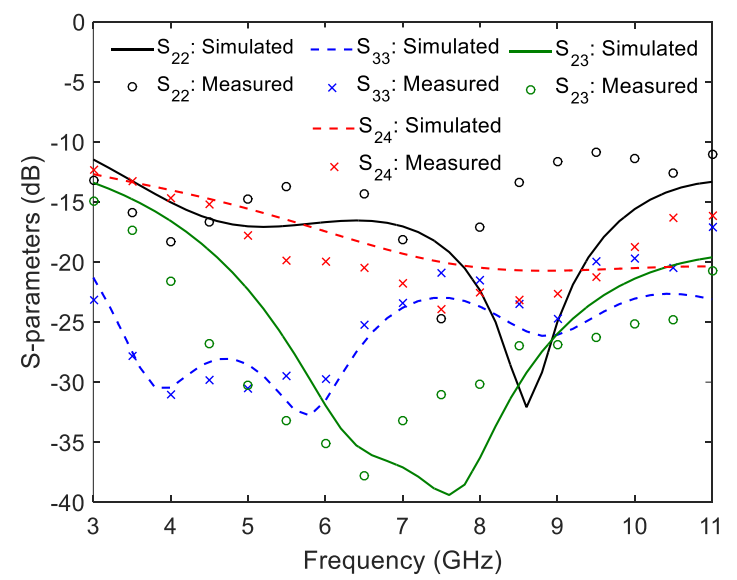

Fig. 7. Simulated and measured $S$-parameters of the proposed divider.

As shown in Fig. 6, simulation and measurement results of the input port matching are below $-12 \mathrm{~dB}$ over the $4-10 \mathrm{GHz}$ band, 
whereas the transmission parameters are equal to $-4.9 \pm 1 \mathrm{~dB}$ over the assigned band. Output ports matching and isolation parameters are in good agreement and are all below $-11 \mathrm{~dB}$ over the assigned band as shown in Fig. 7. The small discrepancies between simulated and measured results are thought to be due to the fabrication process and measurement errors.

Measured group delays of the fabricated divider are depicted in Fig. 8. Measured results show almost a constant response of $0.18 \mathrm{~ns}$ for both $S_{21}$ and $S_{31}$ over the 4-10 GHz band (group delay of $S_{41}=$ group delay of $S_{21}$ due to structural symmetry).

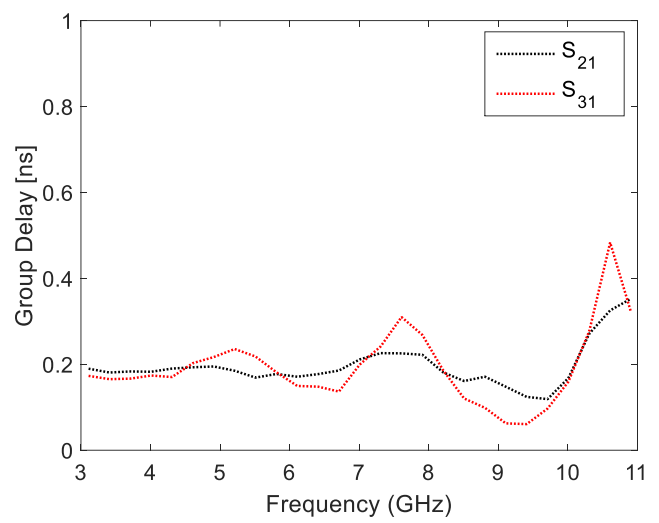

Fig. 8. Measured group delays of the proposed 3-way divider.

Figure 9 shows the measured magnitude and phase imbalances for the proposed equal-split in-phase divider. Measured magnitude imbalance equals to $\pm 0.37 \mathrm{~dB}$, whereas the phase imbalance is $\pm 5.4^{\circ}$ over the design bandwidth. Such results indicate an excellent symmetry of the fabricated layout and hence the proposed device can operate as a power combiner as desired.

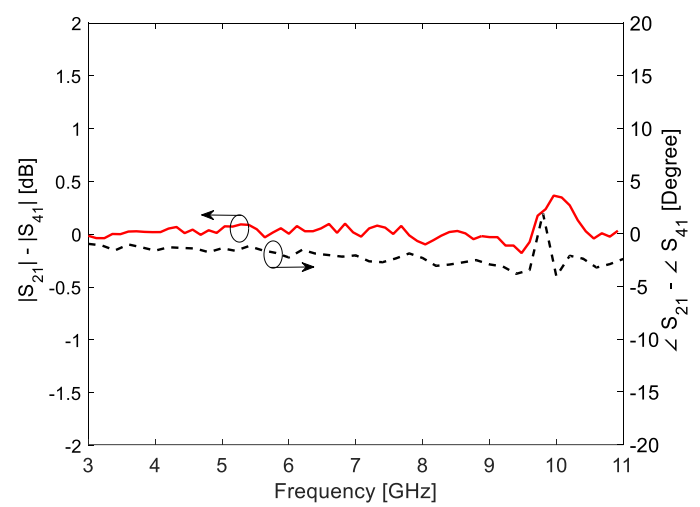

Fig. 9. Measured magnitude and phase imbalances of the proposed 3-way divider.

\section{CONCLUSION}

This paper presented a general design of $N$-way wideband power divider/combiner that can be used in PAs which are a fundamental component in a variety of wireless communication systems. A combining technique was used to reduce the $N$-way divider to its equivalent 2-way model. Then, the even and odd mode analysis were used to obtain the NTLs and the optimized values of the isolation resistors, respectively. For verification purposes, 3-way power divider/combiner with fractional bandwidth of $86 \%$ was fabricated and measured. The good agreement between both simulation and measurement results over the assigned frequency band proves the validity of the design procedure.

\section{REFERENCES}

[1] V. Bridier, D. Ducatteau, M. Olivier, H.-J. Simon, F. Graux, P. Eudeline, and G. Dambrine, "Nonlinear measurement dedicated to non periodic pulse train for radar power amplifier characterization," 2014 IEEE MTT-S Int. Microwave Symp., pp. 1-4, June 2014.

[2] S.J.C.H. Theeuwen, H. Mollee, "S-band radar LDMOS transistors," EuMIC 2009 Europ. Microwave Integ. Cir. Conf., pp. 53-56, Sept. 2009.

[3] T. Munehiro, J. Kobayashi, J. Matsuoka, M. Yoshida, S. Kainuma, and Y. Ueda, "Development of an X-band $800 \mathrm{~W}$ pulsed mini-TWT for active phased array radar modules," IEEE Int. Vacuum Electro. Conf., pp. 99-100, April 2014.

[4] A. C. Paolella, "A new high efficiency, high power transmitter system for wireless communications," MILCOM 2005 IEEE Military Comm. Conf., vol. 2, pp. 1309-1313, Oct. 2005.

[5] L. Lin, L. Zhou, Y. Zhu, Y. Hua, J.-F. Mao, W.-Y. Yin, "Improvement in cavity and model designs of LDMOS power amplifier for suppressing metallic shielding cover effects," IEEE Trans. Electromag. Compatibil., vol. 58. no. 5, pp. 1617-1628, May 2016.

[6] R. N. Simons, E. G. Wintucky, and C. T. Chevalier, "Highefficiency Ka-band waveguide two-way asymmetric power combiner," Int. J. High Speed Electron. and systems, vol. 20, no. 03, pp. 405-415, September 2011.

[7] E. Wilkinson, “An N-way hybrid power divider," IEEE Trans Microwave Theory Tech, vol. 8, no. 1, pp. 116-118, January 1960.

[8] S. C. Cripps, RF Power Amplifiers for Wireless Communications, Artech House, 2006.

[9] A. A. M. Saleh, "Planar electrically symmetric n-way hybrid power dividers/combiners," IEEE Trans Microwave Theory Tech, vol. 28, no. 6, pp. 555-563, Jun 1980.

[10] D. Hawatmeh, K. Al Shamaileh, N. Dib, and A. Sheta, "Design and analysis of a 3-way unequal split ultra-wideband Wilkinson power divider," Int. J. Electronics, vol. 100, no. 8, pp. 1062-1071, Oct. 2012.

[11] J. Zhou, K. A. Morris, and M. J. Lancaster, "General design of multiway multisection power dividers by interconnecting twoway dividers," IEEE Trans Microwave Theory Tech, vol. 55, no. 10, pp. 2208-2215, Oct. 2007.

[12] A. Wentzel, V. Subramanian, A. Sayed, and G. Boeck, "Novel broadband Wilkinson power combiner," $200636^{\text {th }}$ European Microwave Conf., pp. 212-215, Sept. 2006.

[13] C. Wang, W. Che, and P. Russer, "High-isolation multiway power dividing/combining network implemented by broadsidecoupling SIW directional couplers," Int. J. RF Microwave Computer-Aided Eng, vol. 19, no. 5, pp. 577-582, Sept 2009.

[14] A. M. Qaroot, and N. I. Dib, "General design of N-way multifrequency unequal split planar Wilkinson power divider," Prog. Electromagnetic Res. C, vol. 14, pp. 115-129, 2010.

[15] D. Pozar, Microwave Engineering, New York: J. Wiley \& Sons, 2005.

[16] K. Alshamaileh, V. Devabhaktuni, and N. Dib, "Impedencevarying broadband $90^{\circ}$ brach-line coupler with arbitrary coupling levels and higher order harmonic suppression," IEEE Trans. Compon. Pack. Manuf. Technol., vol. 5, no. 10, pp. 1507-1515, Oct. 2015.

[17] K. Shamaileh, M. Almalkawi, V. Devabhaktuni, N. Dib, B. Henin, and A.Abbosh "Fourier-based transmission line ultra-wideband Wilkinson power divider for EARS applications," 2013 IEEE $56^{\text {th }}$ Int. Midwest Symp. Circuits Syst., pp. 872-875, Aug. 2013. 